\title{
SN 1987a: THEORETICAL CONSIDERATIONS
}

\author{
S. E. Woosley \\ Board of Studies in Astronomy and Astrophysics \\ Lick Observatory, University of California at Santa Cruz \\ Santa Cruz CA 95064
}

\section{THE STAR THAT EXPLODED}

There is now general agreement that the presupernova star for 1987a was indeed SK69-202, a star that had visual magnitude 12.36 (Walborn et al. 1987), bolometric correction $1.15^{m}$ (Humphreys and McElroy 1984), and extinction $0.5^{m}$ (Woosley et al. 1987). The distance modulus to the LMC is presently controversial, but most astronomers prefer a value near 18.5 (e.g., Walker 1985; Chiosi and Pigatto 1986) with an uncertainty of perhaps $0.2^{m}$. Putting it together, one arrives at a bolometric magnitude for SK-69-202 of -7.8 with a probable range -7.5 to -8.2 , or a luminosity of $4 \times 10^{38} \mathrm{erg} \mathrm{s}^{-1}$ with a range of 3 to $6 \times 10^{38} \mathrm{erg} \mathrm{s}^{-1}$. At the time of the supernova the hydrogen burning shell contributes negligible energy generation so the critical quantity determining the luminosity is the helium core mass. Inspection of a variety of current stellar models indicates that SK-69-202 had a helium core mass of $6 \pm 1 \mathrm{M}_{\odot}$ from which we may infer that, on the main sequence, it had a mass of $19 \pm 3 \mathrm{M}_{\odot}$. A B3-I supergiant has a surface temperature of $\sim 16,000 \mathrm{~K}$ (Humphreys and McElroy 1984). For the above range of luminosities and assuming a temperature in the range 15,000 to $18,000 \mathrm{~K}$ one obtains a radius for SK-202-69 of $3 \pm 1 \times 10^{12} \mathrm{~cm}$.

Unfortunately observations of the presupernova star do not constrain the mass of the hydrogen envelope. An unknown amount of mass loss could have occurred leaving anywhere from $14 \mathrm{M}_{\odot}$ to as little as a few tenths $\mathrm{M}_{\odot}$. Since the envelope mass greatly affects the dynamics of the explosion, the light curve, and the spectroscopic history of the supernova, its determination is of high priority. Based upon observations of the supernova one conclusion of this paper will be that the envelope mass was in the range 5 to $10 \mathrm{M}_{\odot}$.

Another major and as yet unresolved issue centers upon precisely why SK-202-69 was a blue supergiant, and not a red one. This issue has been recently reviewed by Woosley (1987) and will be briefly summarized here. The essential problem is that there exist multiple solutions to the structure equations for the stellar atmosphere (see also Wheeler, this volume). Two stars having the same helium core mass and only slightly different luminosities, for example, can have radically different envelope structures, either a convective red supergiant or one that is radiative and blue (Woosley, Pinto, and Ensman 1987). There are several physical parameters that may break this symmetry and cause the star to chose one solution and not the other. Among them are metallicity, (extreme) mass loss, and the theory of convection used in calculating the stellar model.

A number of groups have invoked the reduced metallicity of the LMC as the probable cause for the small radius and provided evolutionary calculations to justify their contention. 
My own contribution to this is in Fig. 1. A major difference with respect to Arnett (1987) and Truran and Weiss (1987), for example, is that their stars never became red supergiants, while the low metallicity models here burned helium as red supergiants and moved back to the blue (as recently as 20,000 years ago) just in time to explode. The reason underlying this different behavior is use of the LeDoux criterion for convective instability in the present models rather than the less restrictive Schwarzschild criterion. Observations of the 30 Doradus region show the existence of many red supergiants. So at least a portion of massive stars in the LMC must evolve to or through that state. Also recent observations of low velocity $\left(V \leqslant 200 \mathrm{~km} \mathrm{~s}^{-1}\right)$ nitrogen-rich gas (Kirshner, this volume), presumably a circumstellar shell, require SK-202-69 itself to have experienced considerable mass loss. This would be easier to understand if the star spent a portion of its life as an extended red supergiant.

An alternative means of obtaining a blue supergiant progenitor, while passing along the way through a red supergiant stage, is extreme mass loss. Well before 1987a it had been recognized that a massive star which lost most of its hydrogen envelope would evolve back from the red to the blue in the HR-diagram, exploding as a blue supergiant or, in the extreme limit of complete hydrogen evaporation, a Wolf-Rayet star (Chiosi and Maeder 1986). Models for SK-202-69 of this sort have been computed by Maeder (1987, see also this volume) and Wood and Faulkner (1987). The problem with these solutions is that so much mass must be lost before the star becomes blue again (less than $1 \mathrm{M}_{\odot}$ remains on the presupernova star). Prior to this year it had been believed that stars would need to be massive than $20 \mathrm{M}_{\odot}$, perhaps $40 \mathrm{M}_{\odot}$ (Humphreys 1984) in order to lose most of their envelope. More importantly, as we shall see, the explosion of a $6 \mathrm{M}_{\odot}$ helium core tamped by less than a few solar masses of envelope will give hydrogenic velocities, an optical light curve, and an $x$-ray light curve incompatible with observations of $1987 \mathrm{a}$.

\section{MODELS}

Because the explosion energy and hydrogen envelope mass are not known ab initio, the strategy here has been to calculate a variety of models based upon the explosion of a 6 $\mathrm{M}_{\odot}$ helium core (extracted from a previous $20 \mathrm{M}_{\odot}$ presupernova model; Woosley and Weaver 1986) capped by envelopes of various masses. The envelopes were constructed separately in thermal and hydrostatic equilibrium with a radius and luminosity appropriate to SDK-20269. Explosion was simulated in the hybrid configuration by removing the collapsed iron core and replacing it with a piston of specified trajectory. Rapid motion of the piston initiated a shock wave that ejected all exterior matter. After a time, when the expansion had become homologous, the total kinetic energy, hereafter referred to as the "explosion energy," could be sampled. A collection of such models is given in Table 1 which gives, besides the explosion energy and envelope mass, the time when the shock wave broke through the surface of the star, $t_{\text {break }}$; the column depth to the center of the star (actually the outer edge of the ${ }^{56} \mathrm{Ni}$ mass) when the supernova was $10^{6} \mathrm{~s}$ old, $\phi_{0}$; how long the energy contained in the hydrogen envelope following shock passage could power the supernova at the observed luminosity, $\tau_{H}$; and the velocity of the slowest moving hydrogen in the ejecta, $v_{\text {slow }}$. This last quantity is a powerful constraint upon the models. Elias and Gregory (1987) have determined that the slowest hydrogen ejected was moving no faster than and probably close to $2100 \mathrm{~km} \mathrm{~s}^{-1}$. This immediately suggests that the favored model will resemble some subset of $3 \mathrm{VL}, 5 \mathrm{~L}, 10 \mathrm{H}$, and $14 \mathrm{VH}$. 


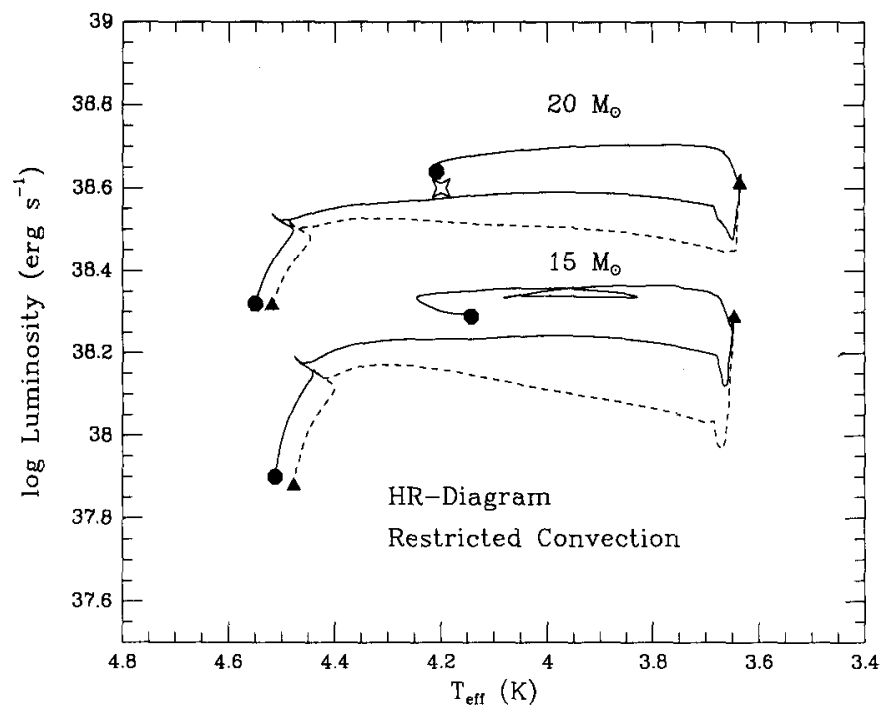

Fig. 1 - Hertzsprung-Russell diagram for stars of 15 and $20 \mathrm{M}_{\odot}$ and composition appropriate to the $\mathrm{LMC}\left(\mathrm{Z}_{\odot} / 4\right)$ (solid lines) and to the sun (dashed lines) evolved through hydrogen, helium, and carbon burning. The location of the presupernova stars are indicated. The four-pointed star indicates the best estimated properties of SK-202-69.

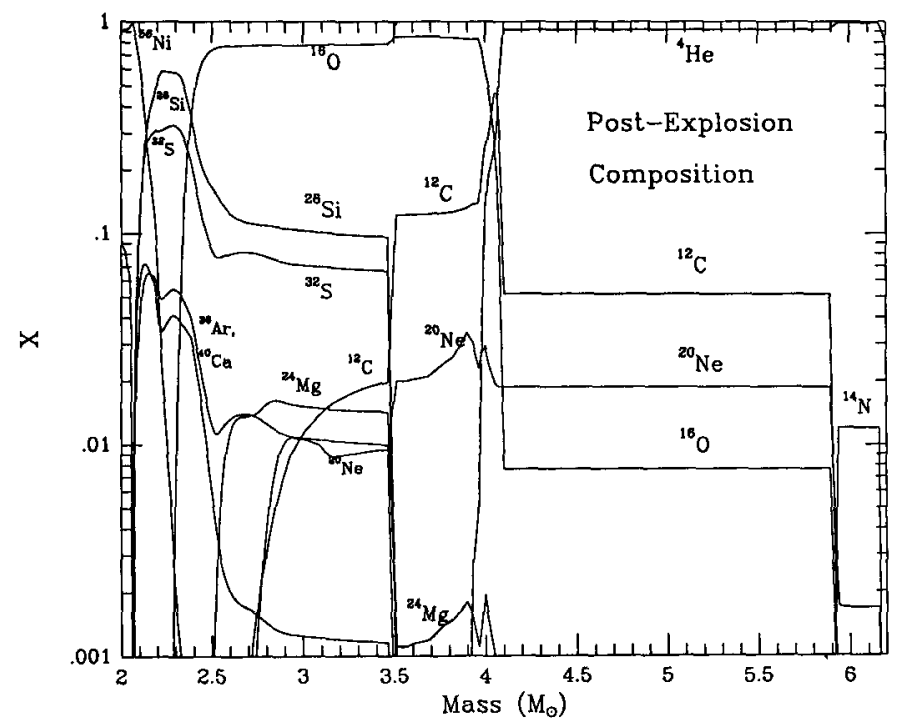

Fig. 2 - Composition of the $6 \mathrm{M}_{\odot}$ helium core used in all studies. Interior to about $3 \mathrm{M}_{\odot}$ the composition is a result of explosive nucleosynthesis. Farther out the fossil remnants of previous burning stages are ejected. 
TABLE 1: MODEL DEFINITIONS

\begin{tabular}{rrrrrrr}
\hline Model & $1 \mathrm{~L}$ & $1 \mathrm{H}$ & $3 \mathrm{~L}$ & $3 \mathrm{H}$ & $5 \mathrm{~L}$ & $5 \mathrm{H}$ \\
\hline Expl. KE $\left(10^{51} \mathrm{erg}\right)$ & 0.65 & 1.4 & 0.65 & 1.4 & 0.65 & 1.3 \\
Envel. Mass $\left(\mathrm{M}_{\odot}\right)$ & 1 & 1 & 3 & 3 & 5 & 5 \\
$\mathrm{t}_{\text {break }}(\mathrm{s})$ & 3100 & 2200 & 5200 & 3700 & 5500 & 3900 \\
$\phi_{o} / 10^{4}\left(\mathrm{~g} \mathrm{~cm}^{-2}\right)$ & 1.4 & 0.52 & 2.3 & 1.0 & 4.6 & 1.9 \\
$\tau_{H}($ days $)$ & 12.5 & 14.7 & 20.5 & 23.4 & 23.9 & 26.7 \\
$v_{\text {slow }}(H) \mathrm{km} \mathrm{s}^{-1}$ & 4000 & 5600 & 2400 & 3500 & 1800 & 2800 \\
\hline \hline Model & $10 \mathrm{~L}$ & $10 \mathrm{H}$ & $14 \mathrm{H}$ & $14 \mathrm{VH}$ & $3 \mathrm{VL}$ & $1 \mathrm{VVL}$ \\
\hline Expl. KE $\left(10^{51} \mathrm{erg}\right)$ & 0.65 & 1.4 & 1.3 & 2.6 & 0.41 & 0.34 \\
Envel. Mass $\left(\mathrm{M}_{\odot}\right)$ & 10 & 10 & 14 & 14 & 3 & 1 \\
$\mathrm{t}_{\text {break }}(\mathrm{s})$ & 9000 & 6400 & 7600 & 5500 & 6300 & 4000 \\
$\phi_{o} / 10^{4}\left(\mathrm{~g} \mathrm{~cm}^{-2}\right)$ & 15.4 & 7.1 & 17.1 & 7.8 & 5.1 & 8.0 \\
$\tau_{H}\left(\right.$ days $\left.^{2}\right)$ & 35.2 & 40.2 & - & - & - & - \\
$v_{\text {slow }}(H) \mathrm{km} \mathrm{s}^{-1}$ & 1100 & 1700 & 1200 & 1700 & 1800 & 2900 \\
\hline
\end{tabular}

The composition within the helium core following shock wave passage is given in Fig.

2.

\section{THE EARLY LIGHT CURVE}

After approximately one minute the shock initiated by iron core collapse arrives at the outer edge of the helium core whose radius is typically $5 \times 10^{10} \mathrm{~cm}$. The hydrodynamic interaction with the envelope slows the helium core down, the deceleration propagating into the core as a "reverse shock." Meanwhile the outgoing shock continues though the hydrogen. The time when the shock breaks through the surface of the envelope can be estimated (Shigeyama et al. 1987; Woosley 1987),

$$
t_{b} \sim 2500\left(\frac{M_{e n v} / M_{\odot}}{E_{51}}\right)^{1 / 2} \mathrm{~s},
$$

with $E_{51}$ the explosion energy in units of $10^{51} \mathrm{erg}$. This result is in very good agreement with the calculated values for shock break out given in Table 1.

As the shock breaks out the electromagnetic display commences (Fig. 3). Initially the temperature is so high $\left(2-3 \times 10^{5} \mathrm{~K}\right)$ that most of the radiation will be in the ultraviolet. Figure 4 shows the comparison between the first two days of optical data and the calculated visual magnitudes for 4 models (Table 1) having a variety of explosion energies and envelope masses. This set of curves was calculated using electron scattering opacity only and a bolometric correction based upon simple, single temperature blackbody model. It is well known, however, that the temperature at the photosphere, or more properly "surface of last scattering", is not a good match to the color temperature in situations where electron scattering opacity dominates. Instead the radiation and electron temperature fall out of equilibrium and the star radiates a dilute blackbody spectrum having a color temperature, $T_{c}$, approximately equal to the local temperature where the generalized optical depth, $\left(\kappa_{t o t} \kappa_{a b s}\right)^{1 / 2} \phi$, is unity. Here $\kappa_{\text {tot }}$ and $\kappa_{a b s}$ are respectively the total opacity (approximately the electron scattering opacity) and that portion of the opacity in which interaction does not preserve photon energy and $\phi$ is column depth. A 


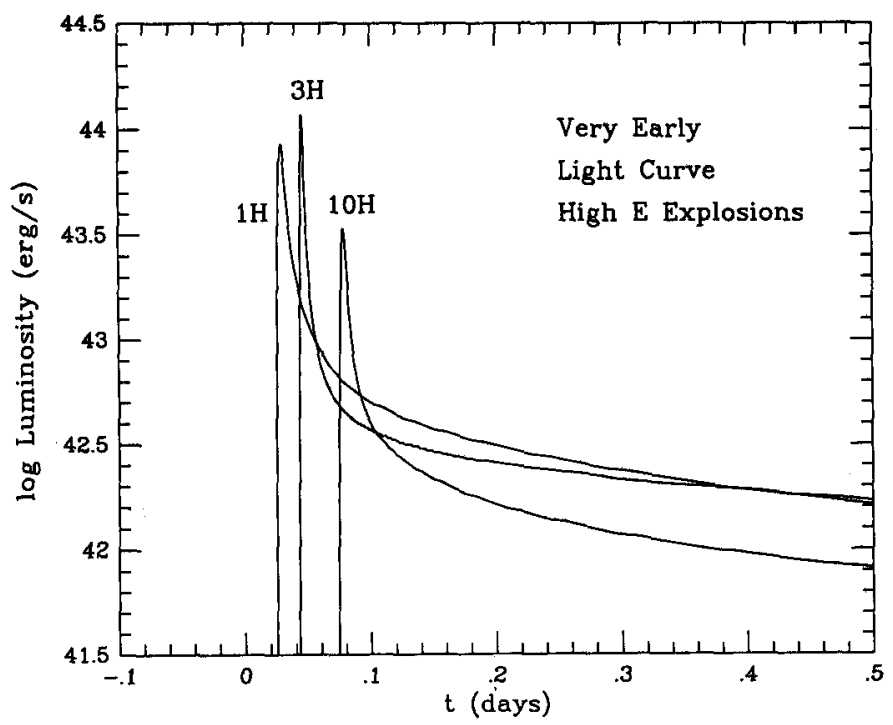

Fig. 3 - Bolometric luminosity during the first half day of several explosions (Table 1). The decline during the first hour is especially rapid. A total of about $10^{47} \mathrm{erg}$ is emitted as hard UV-radiation during the first day of the supernova.

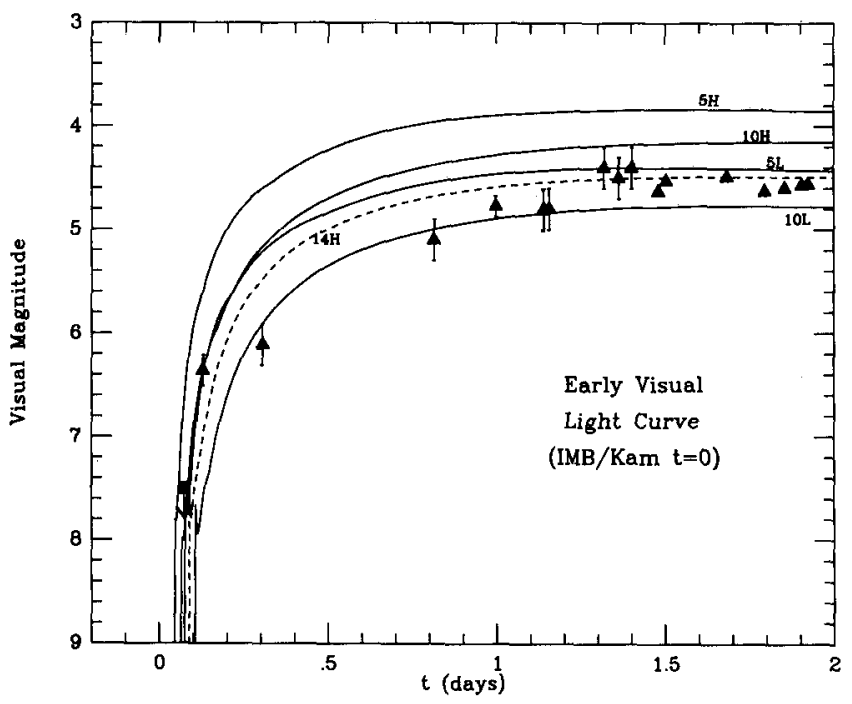

Fig. 4 - Visual light curve during the first two days. Time zero is defined by the Kamiokande - IMB neutrino signal. Light curves from four models (Table 1) are shown as solid lines. A distance modulus of 18.5 and visual extinction of $0.5^{m}$ have been adopted. Shown for comparison are observational data points. 
photon originating from deeper in the supernova than this will undergo enough scattering events to encounter several energy non-conserving events and be (approximately) thermalized. On the other hand the radiative diffusion equation may be solved for a region of constant luminosity to yield $\left(T_{c} / T_{e}\right)^{4}=\tau_{e}$ with $T_{e}$ the effective emission temperature at the scattering photosphere (what the code edits) and $T_{c}$ the temperature at electron scattering optical depth $\tau_{e}$. Combining equations one finds that the color temperature will be approximately $\left(\kappa_{t o t} / \kappa_{a b s}\right)^{1 / 8}$ times the effective emission temperature evaluated at the scattering photosphere. The problem then is one of determining the non-conservative opacity as a function of temperature, density, composition, and velocity shear.

The relatively weak dependence on the ratio $\kappa_{t o t} / \kappa_{a b s}$ suggests that the modification to our calculated results will not be great except at very early times. The effective temperature calculated for Model $10 \mathrm{H}$, for example, is, without modification, within $15 \%$ of the values inferred from the spectrum (Suntzeff, private communication) on days $1.14(13,600 \mathrm{~K}), 1.51$ $(12,700 \mathrm{~K})$, and $1.85(11,690 \mathrm{~K})$. Figure 5 illustrates the effect for $\kappa_{t o t} / \kappa_{a b s}=1,0.3$, and 0.1 . The latter corresponds to a color temperature one third greater than the effective emission temperature. Karp et al. (1977) have considered the effect of Doppler broadened lines on the bound-bound opacity. For typical photospheric densities $\left(10^{12} \mathrm{~g} \mathrm{~cm}^{-3}\right)$ and temperatures $(5000 \mathrm{~K}$ to $50,000 \mathrm{~K})$ the line opacity is approximately $20 \%$ to $200 \%$ that of electron scattering (see their Table 3). This should keep the color temperature within about $20 \%$ of the effective emission temperature.

\section{THE LATER LIGHT CURVE}

Figure 6 shows the bolometric light curves for the first 200 days for 4 of the models defined in Table 1. Following shock break out and its associated high temperatures, the supernova enters a "plateau" stage, which lasts about one month, with energy released (though not provided) by recombination. For greater explosion energies, larger presupernova radii, and smaller hydrogen masses the initial light curve is brighter. This reflects both the greater internal energy deposited in the envelope by a more energetic explosion and the greater expansion velocity given an envelope having lower mass. The duration of this plateau ( $\tau_{H}$ in Table 1$)$ is also determined by the explosion energy and envelope mass.

Following hydrogen recombination the luminosity rises at a rate that is very sensitive to the explosion energy, the envelope mass, and to the opacity in the helium core. The ultimate source of the energy here is the decay of ${ }^{56} \mathrm{Co}$ to ${ }^{56} \mathrm{Fe}$, a reaction that has powered the light curve since late March and especially through the peak and tail. Because the amount of ${ }^{56} \mathrm{Ni}$ synthesized is artificially constrained to be the same $\left(0.07 \mathrm{M}_{\odot} ; \S \mathrm{V}\right)$ in all our models, the peak luminosity does not vary greatly with envelope mass in Fig. 6. In a more realistic case the ${ }^{56} \mathrm{Ni}$ mass would depend upon the explosion energy and would be greater for more energetic explosions. We see from Fig. 6 that the favored model, for an explosion energy near $1.4 \times 10^{51}$ erg, has about $10 \mathrm{M}_{\odot}$ of envelope, consistent with Fig. 4 and restrictions on the slowest moving hydrogen (§II).

All of the light curves in Fig. 6, however, suffer from an obvious deficiency - they go through a period of decline near the end of hydrogen recombination that is not reflected in the observations. Indeed, quite the opposite is observed - after the first week the light curve increases, steadily and smoothly, all the way to its peak. This smoothness of the bolometric light curve has been one of the most perplexing aspects of 1987a. In Fig. 7 Model 10H has been recalculated using i) an opacity, in addition to electron scattering, of $0.001 \mathrm{~cm}^{2} \mathrm{~g}^{-1}$ for 


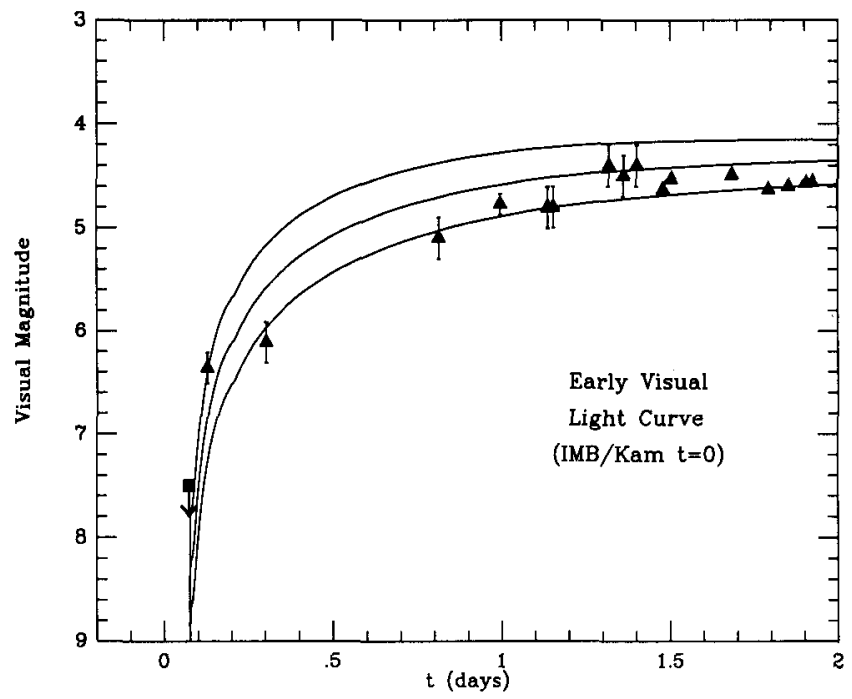

Fig. 5 - Correction to the early visual light curve of Model $10 \mathrm{H}$ for the fact that the color temperature does not equal the effective emission temperature for an atmosphere whose opacity is dominantly due to electron scattering. The three curves from top to bottom have the nonconservative opacity equal to $1,0.3$, and 0.1 of the electron scattering opacity.

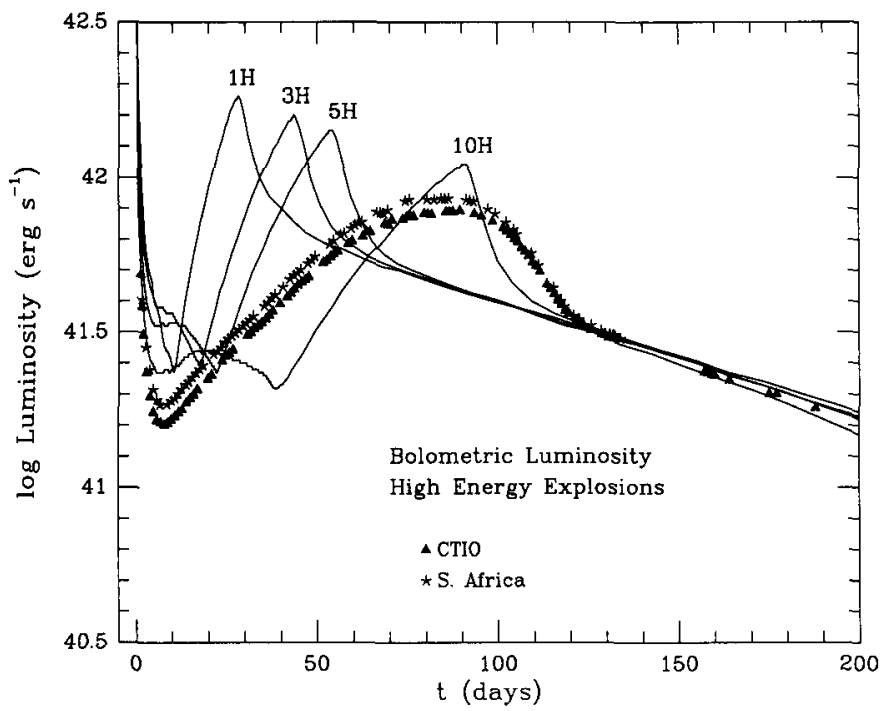

Fig. 6 - Bolometric luminosities during the first 200 days for 4 of the models defined in Table 1 compared to data from Catchpole et al. (1987) and Hamuy et al. (1987). All models employed the same $6 \mathrm{M}_{\odot}$ helium core capped by hydrogen envelopes of various masses. The opacity, chiefly due to electron scattering while the gas remains ionized, was given a lower floor of $0.02 \mathrm{~cm}^{2} \mathrm{~g}^{-1}$ for elements heavier than helium. 
both hydrogen and helium in the envelope; ii) an artificial gradient of hydrogen and helium in the envelope (but no mixing in the core); and iii) an additive opacity of $0.025 \mathrm{~cm}^{2} \mathrm{~g}^{-1}$ within the helium core (internal to $6.2 \mathrm{M}_{\odot}$ ). Effect $\mathrm{i}$ ) reduces the light curve during the first $\sim 10$ days compared to Fig. 6 and relates physically to the opacity of broadened lines. The value employed is probably less than what actually characterizes the outer layers with large velocity shear. Effect ii) is most important and reflects, partly, the real gradient of helium and hydrogen that would be present in the envelope and mixing that would have occurred during the explosion. Effect iii), the additive opacity in the core, affects the width and timing of the light curve peak and is taken to represent the Doppler-broadened line opacity of helium and heavy elements in the core. The good agreement shows that the observations can be fit very well by reasonable modifications to the simple model. Also shown in Fig. 7 are two light curves that used the same parameters but zero or twice as much radioactivity. Both are clearly excluded by the observations. In the case of zero radioactivity the light curve dies at approximately $\tau_{H}$ for Model $10 \mathrm{H}$.

\section{THE RADIOACTIVE TAIL AND THE NICKEL BUBBLE}

Unlike Type Ia supernovae, the slow expansion of the core of Type II's renders them optically thick to $\gamma$-radiation for a period of about 2 years. During the first year at least this $\gamma$-radiation is degraded chiefly into optical and ultraviolet radiation which, after the peak of the light curve, diffuses out in a time short compared to the elapsed time. During this interval the UV-optical light curve should track exactly the ${ }^{56}$ Co decay rate. Figure 8 shows the bolometric luminosity of Catchpole et al. (1987) and Hamuy et al. (1987) during the first 188 days based upon the same assumptions regarding distance modulus and visual extinction as the authors (18.5 and $0.6^{m}$ respectively). Also given are two lines generated by the equation

$$
\dot{S}_{n u c}=3.90 \times 10^{10} e^{-t / \tau_{N i}}+7.21 \times 10^{9}\left(e^{-t / \tau_{C o}}-e^{-t / \tau_{N i}}\right) \mathrm{erg} \mathrm{g}^{-1} \mathrm{~s}^{-1}
$$

multiplied by $0.07 \mathrm{M}_{\odot}$ and $0.2 \mathrm{M}_{\odot}$ respectively of radioactive ${ }^{56} \mathrm{Ni}$ produced initially in the explosion. It is apparent that very nearly $0.07 M_{\odot}$ of mass 56 has been produced in the explosion, though a different distance modulus or correction for visual extinction would give a slightly different value. Because of the certainty with which it can be determined this value of ${ }^{56}$ Co mass was employed in all calculations reported in this paper.

As an interesting aside, we note that the near match of the bolometric light curve on the tail to that which would be provided by ${ }^{56} \mathrm{Co}$ (eq. 2) places limits on the possible contribution from a pulsar. In particular on day 188 the bolometric luminosity of the supernova was $1.8 \times 10^{41}$ $\mathrm{erg} \mathrm{s}^{-1}$ (Nick Suntzeff, private communication). The contribution of a second source having a very different mean life would have led to discernable deviations from eq. (2) if the background source contributed more than a small fraction of this, say $10 \%$. If a pulsar exists, similar to the one in the Crab Nebula, its luminosity at this stage would be $L \sim 4 \times 10^{43} B_{12}^{2} P_{o}^{-4} \mathrm{erg} \mathrm{s}^{-1}$ with $B_{12}$ the field strength in units of $10^{12}$ gauss and $P_{o}$ the period in ms (Ostriker and Gunn 1969). Limiting $L$ to $\sim 2 \times 10^{40} \mathrm{erg} \mathrm{s}^{-1}$ thus implies (for $B_{12}=4.3$ as in the Crab; Manchester and Taylor 1977), that the period of the pulsar is presently greater than $14 \mathrm{~ms}$ (i.e., $1 / 2$ the Crab).

The energy released by the decay of ${ }^{56} \mathrm{Ni}\left(2.96 \times 10^{16} \mathrm{erg} \mathrm{g}^{-1}\right)$ and by ${ }^{56} \mathrm{Co}\left(6.41 \times 10^{16}\right.$ erg $\left.\mathrm{g}^{-1}\right)$ is comparable to the kinetic energy density in the iron and overlying layers. Following the passage of the reverse shock, all of the elements heavier than helium are typically moving at 1000 to $2000 \mathrm{~km} \mathrm{~s}^{-1}$ corresponding to a kinetic energy density of $\sim 2 \times 10^{16} \mathrm{erg}^{-1}$. Even 


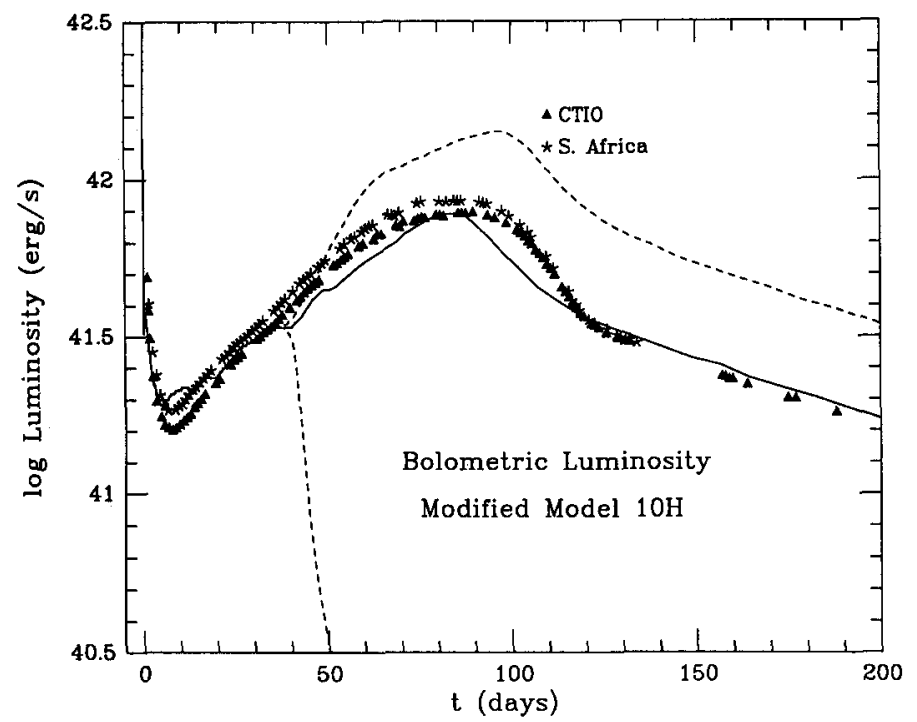

Fig. 7 - Comparison of the observed bolometric light curve to several modified versions of Model 10H. Each employed an artificial gradient of helium in the envelope to aid in replicating the rising nature of the light curve during the first 40 days. Each also adopted a floor to the hydrogen and helium opacities in the envelope of $0.001 \mathrm{~cm}^{2} \mathrm{~g}^{-1}$ and to the opacity within the helium core of $0.025 \mathrm{~cm}^{2} \mathrm{~g}^{-1}$ (including helium itself). The two dashed lines show the effect of varying the mass of ${ }^{56} \mathrm{Co}$ produced in the explosion. The upper curve used twice as much ${ }^{56} \mathrm{Ni}$ $\left(0.14 \mathrm{M}_{\odot}\right)$; the lower curve, no radioactivity.

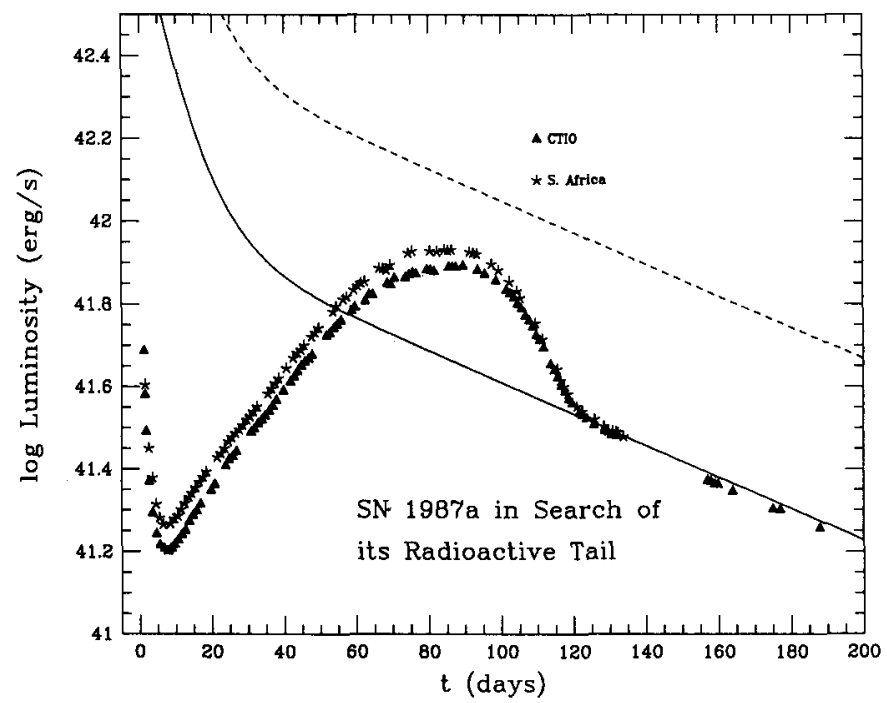

Fig. 8 - The observed bolometric light curve (Catchpole et al. 1987 and Hamuy et al. 1987) compared to that which would result from $100 \%$ optical conversion and escape of energy from the radioactive decay of ${ }^{56} \mathrm{Ni}$ and ${ }^{56} \mathrm{Co}$. The upper curve is for $0.20 \mathrm{M}_{\odot}$ of mass 56 and the lower curve is for $0.07 \mathrm{M}_{\odot}$. 
more relevant is the fact that the velocity shear across the region of heavy elements is only a few hundred $\mathrm{km} \mathrm{s}^{-1}$. Thus the decay of ${ }^{56} \mathrm{Ni}$ and ${ }^{56} \mathrm{Co}$ has dynamic consequences. Of great potential significance is the fact that, in one dimensional calculations, the decay of ${ }^{56} \mathrm{Co}$ at the center of the supernova produces a density inversion that is Rayleigh Taylor unstable. It is thus likely that when considered in multiple dimensions the bubble may "pop," that is develop "fingers" that lead to the mixing of ${ }^{56} \mathrm{Co}$ out into the helium core. This mixing might also be accompanied by clumping. If voids develop in the heavy elements overlying the core, this might allow us to see the $\mathrm{x}$-rays and $\gamma$-rays from the ${ }^{56} \mathrm{Co}$ decay, as well as any manifestations of the central neutron star, somewhat earlier than the simple one-dimensional models would predict.

\section{X-RAY AND GAMMA-LINE FLUXES}

The only species which will emit $\gamma$-line radiation at a level that might possibly be detected in the near future is ${ }^{56} \mathrm{Co}$. The flux from a mass, $M_{56}$ of ${ }^{56} \mathrm{Co}$ in solar masses located in the $\mathrm{LMC}(50 \mathrm{kpc})$ is

$$
F=0.602\left(\frac{M_{56}}{0.1 M_{\odot}}\right) \exp \left(-t / 113.6 \mathrm{~d}-\kappa_{\gamma} \phi_{o}\left(t_{o} / t\right)^{2}\right) \mathrm{cm}^{-2} \mathrm{~s}^{-1}
$$

where $t$ is the elapsed time since the explosion, $t_{o}$ some fiducial time at which the column depth to the edge of the ${ }^{56} \mathrm{Co}$ layer, $\phi_{o}$, is to be determined, and $\kappa_{\gamma}$ is the opacity to $1 \mathrm{MeV}$ $\gamma$-rays. Here $F$ is the flux of some line, such as $847 \mathrm{keV}$, through which all decays proceed and homologous expansion has been assumed. An appropriate value of $\kappa_{\gamma}$ is $0.06 \mathrm{~cm}^{2} \mathrm{~g}^{-1}$ and a reasonable time to evaluate the column depth is $t_{o}=10^{6} \mathrm{~s}$ (Table 1).

This flux will have a maximum at time

$$
t_{\max }=\left(2 \tau_{C o} \kappa_{\gamma} \phi_{o} t_{o}^{2}\right)^{1 / 3}=263\left(\phi_{o} / 10^{4}\right)^{1 / 3} \text { days. }
$$

The maximum flux for models in Table 1 is easily obtained by evaluating eq. (3) at time $t=t_{\max }$,

$$
\begin{aligned}
F_{\text {max }} & =0.602\left(\frac{M_{56}}{0.10 M_{\odot}}\right) \exp \left[3\left(\frac{\kappa_{\gamma} t_{o}^{2} \phi_{o}}{4 \tau_{C o}^{2}}\right)^{1 / 3}\right] \\
& =0.602\left(\frac{M_{56}}{0.10 M_{\odot}}\right) \exp \left(-0.161 \phi_{o}^{1 / 3}\right) \mathrm{cm}^{-2} \mathrm{~s}^{-1},
\end{aligned}
$$

a result which is extremely sensitive to the column depth at $t_{o}$, i.e., to the expansion rate. Models 3VL, 5L, and $10 \mathrm{H}$, which have $\phi_{0} / 10^{4} \mathrm{~g} \mathrm{~cm}^{-2}=5.1,4.6$, and 7.1 respectively would have peak fluxes of $1.1,1.4$, and $0.5 \times 10^{-3} \mathrm{~cm}^{-2} \mathrm{~s}^{-1}$ at days 450,440 , and 510 respectively. Model $1 \mathrm{H}$, on the other hand, which unfortunately (for $\gamma$-line astronomers) is disallowed by comparison to the light curve and photospheric velocity history, would have presented a flux of about $0.026 \mathrm{~cm}^{-2} \mathrm{~s}^{-1}$ on day 210 .

The $\gamma$-ray optical depth at maximum emission is $1.4\left(\phi_{o} / 10^{4} \mathrm{~g} \mathrm{~cm}^{-2}\right)^{1 / 3}$, which is in the range 2-3 for any reasonable model. Thus the continuum will be quite strong. Indeed well before the $\gamma$-lines become visible at a detectable level, the supernova should be quite bright in hard $\mathrm{x}$-rays. At the meeting Tanaka (see this volume) announced the discovery of the supernova in the 10 to $30 \mathrm{keV}$ band by GINGA beginning in mid-August. Prior to the meeting Pinto and $I$ had calculated the expected $\mathrm{x}$-ray spectrum and light curve for Model $10 \mathrm{H}$ (as had several other groups). Without mixing or clumping, the $\mathrm{x}$-rays would not have been detectable until 

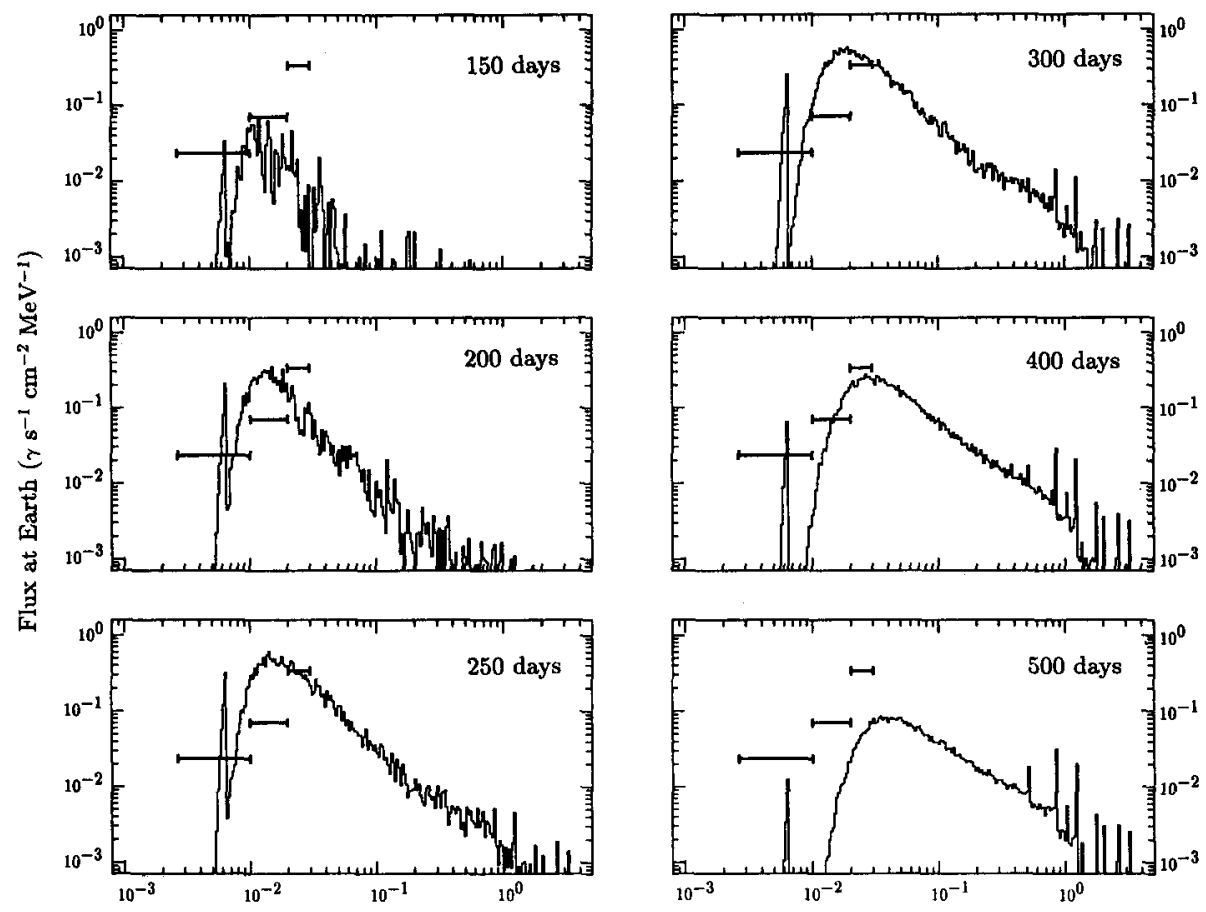

Energy $(\mathrm{MeV})$

Figure 9. X-ray spectra at several times for Model 5LM evaluated at the dates shown from Monte Carlo calculations by Pinto and Woosley (1987). Horizontal error bars show the GINGA sensitivity at various energies.

about day 250 (Nov. 1), i.e., a discrepancy of about 75 days. Immediately upon returning home we calculated the expected light curve of a more promising model, $5 \mathrm{~L}$, in which some outward mixing of the ${ }^{56} \mathrm{Co}$ was presumed to have occurred ( $(\mathrm{V})$. In particular Model 5LM (Woosley 1987) was examined in which the ${ }^{56} \mathrm{Co}$ abundance was artificially given a gradient such that it declined smoothly by a factor of 10 between the center of the supernova and the edge of the helium and was zero in the hydrogen envelope. The x-ray spectra and onset (Fig. 9) are in reasonable accord (perhaps bright by a factor of two and a little too soft a spectrum) with the data available in September. For further detail see Pinto and Woosley (1987).

\section{SUMMARY}

The star that exploded, SK-202-69, was, as theory required, a massive star. When it lived on the main sequence, it had a mass of $19 \pm 3 \mathrm{M}_{\odot}$. At the time it exploded it had a helium core mass of $6 \pm 1 \mathrm{M}_{\odot}$, a radius $3 \pm 1 \times 10^{12} \mathrm{~cm}$, a luminosity 3 to $6 \times 10^{38} \mathrm{erg} \mathrm{s}^{-1}$, and an effective temperature 15,000 to $18,000 \mathrm{~K}$. Further consideration of the stellar models (Woosley 1987; Nomoto, this volume) suggests that the iron core mass at the time of collapse was $1.45 \pm 0.15 \mathrm{M}_{\odot}$. Adding $\sim 0.15 \mathrm{M}_{\odot}$ for matter between the iron core and the entropy jump 
which usually demarks the "mass cut" and subtracting $10 \%$ for the binding energy implies a gravitational mass for the collapsed remnant of $1.40 \pm 0.15 \mathrm{M}_{\odot}$. In the particular case of a 20 $\mathrm{M}_{\odot}$ star having an entropy jump at $1.55 \mathrm{M}_{\odot}$ (Woosley and Weaver 1987) where the "mass cut" is most likely to develop (see also Nomoto et al. 1987), the remnant would have gravitational mass very nearly $1.40 \mathrm{M}_{\odot}$. This compares favorably with the accurate mass determined for two neutron stars in binary pulsar PSR $1913+16\left(1.451 \pm 0.007 \mathrm{M}_{\odot}\right.$ and $1.378 \pm 0.007 \mathrm{M}_{\odot}$; Taylor 1986) which are believed to be the remnants of stars in the 16 to $18 \mathrm{M}_{\odot}$ range (Burrows and Woosley 1986). Thus the object is almost certainly a neutron star, not a black hole. However, even if it is a pulsar, radiation from this neutron star has not contributed significantly to the light curve thus far. Therefore if it has a magnetic moment like that of the Crab pulsar and accretion has not choked the emission mechanism, the neutron star must be rotating with a period longer than $15 \mathrm{~ms}$.

The explosion mechanism itself might have been due to the shock wave created by core bounce, especially for iron core masses in the lower range of the error bars, but more likely required the aid of neutrino energy transport, i.e., was a delayed explosion. This latter alternative is more consistent with properties of the neutrino burst measured by Kamiokande and IMB (Mayle and Wilson 1987). A severe constraint on both the presupernova structure and the explosion mechanism (that has yet to be imposed and explored) is that the explosion eject $0.07 \mathrm{M}_{\odot}$ of ${ }^{56} \mathrm{Ni}$. Decay of this ${ }^{56} \mathrm{Ni}$ and its daughter ${ }^{56} \mathrm{Co}$ releases sufficient energy that mixing of the heavy elements (carbon through calcium) may have occurred following the explosion. Rayleigh-Taylor instability in the reverse shock (e.g., Chevalier and Klein 1978) may also have led to mixing. Models having mixed compositions agree marginally better with observations and may be necessary to understand the $\mathrm{x}$-ray light curve.

In any case the total kinetic energy of the explosion did not exceed $2 \times 10^{51} \mathrm{erg}$, and even this large a value is only allowed if the star had not lost much of its hydrogen envelope prior to exploding. Otherwise the early light curve would have been too bright (Fig. 4), the radioactive portion of the light curve would have peaked too early (Fig. 6), and the slowest hydrogen ejected would have had a velocity in excess of $2100 \mathrm{~km} \mathrm{~s}^{-1}$ (Table 1; Woosley 1987) which is disallowed by observations (Elias and Gregory 1987). For explosion energies less than about $3 \times 10^{50} \mathrm{erg}$, on the other hand, large portions of the star would have failed to achieve escape velocity, especially in the case of large envelope masses which effectively tamp the expansion of the heavy element core. We know that this did not occur because of the radioactivity that is now powering the light curve. Even for energies as low as $4 \times 10^{50} \mathrm{erg}$ the envelope mass cannot be less than about $3 \mathrm{M}_{\odot}$ or the light curve would have risen too rapidly and peaked too early . Putting it together we obtain Fig. 10 which summarizes the allowed range of explosion energy and envelope mass allowed by constraints coming from the light curve. The favored model emerges as one having a hydrogen envelope in the range 5 to $10 \mathrm{M}_{\odot}$ and thus an explosion energy in the range 0.8 to $1.5 \times 10^{51} \mathrm{erg}$. 


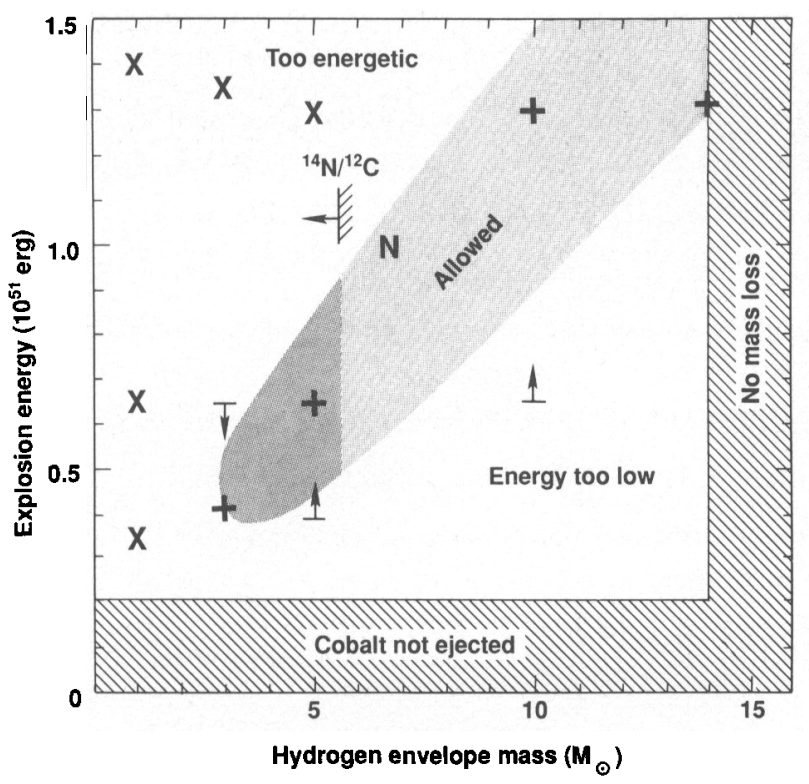

Figure 10. Allowed values of explosion energy and hydrogen envelope mass are broadly delineated for 1987a. Based upon the explosion of a $6 \mathrm{M}_{\odot}$ core (main sequnce mass $20 \mathrm{M}_{\odot}$ ), the atmosphere can be no greater than $14 \mathrm{M}_{\odot}$. Symbols " $\mathrm{X}$ " denote a model that can be excluded on the basis of one or more observational constraints; " + " indicates a moderately successful model; arrows indicate lower and upper bounds provided by three of the models; and " $\mathrm{N}$ " a successful model recently published by Nomoto et al (1987). Explosion energies below $3 \times 10^{50}$ erg lead to reimplosion of the core and loss of all ${ }^{56} \mathrm{Co}$.

This work has been supported by the National Science Foundation (AST-84-18185). I would like to express my appreciation to Ken Nomoto for his hospitality and for his role in organizing a productive and unually exciting meeting.

\section{BIBLIOGRAPHY}

Arnett, W. D. 1987, Ap. J., 319, 136.

Burrows, A., and Woosley, S. E. 1986, Ap. J., 308, 680.

Catchpole, R. M., Menzies, J. W., Monk, A. S., Wargau, W. F. and 16 others. 1987, preprint, South Africa Observatory..

Chevalier, R., and Klein, R. I. 1978, Ap. J., 219, 994.

Chiosi, A., and Maeder, A. 1986, Ann. Rev. Astron. and Ap., 24, 329.

Chiosi, C., and Pigatto, L. 1986, Ap. J., 308, 1.

Elias, and Gregory 1987, in preparation for Ap. J. 
Hamuy, M., Suntzeff, N. B., Gonzalez, R., and Martin, G. 1987, NOAO Preprint No. 102, submitted to $A p$. $J$.

Humphreys, R. M. 1984, in Observational Tests of Stellar Evolution Theory, IAU Symposium 105, Ed. A. Maeder and A. Renzini, (D. Reidel: Dordrecht), p. 279.

Humphreys, R. M., and McElroy, D. B. 1984, Ap. J., 284, 565.

Karp, A. H., Lasher, G., Chan, K. L., and Salpeter, E. E. 1977, Ap. J., 214, 161.

Maeder, A. 1987, preprint to appear in Proc. of ESO Workshop on SN1987a, ed. J. Danziger, in press.

Manchester, R. N., and Taylor, J. H. 1977, Pulsars, (Freeman: San Francisco).

Mayle, R. W., and Wilson, J. R. 1987, preprint, submitted to Ap. J.

Nomoto, K., Shigeyama, T., and Hashimoto, K. 1987, in Proc. ESO Workshop. on SN1987a, ed. J. Danziger, in press.

Ostriker, J. P., and Gunn, J. E. 1969, Ap. J., 157, 1395.

Pinto, P. A., and Woosley, S. E. 1987, submitted to Ap. J.

Shigeyama, T., Nomoto, K., Hashimoto, K., and Sugimoto, D. 1987, Nature, 328, 320.

Taylor, J. 1986 in The Origin and Evolution of Neutron Stars, ed. D. J. Helfand and J.-H. Huang, (D. Reidel: Dordrecht), p. 383.

Truran, J. W., and Weiss, A. 1987, Max Planck Inst. for Ap. preprint No. 303. To appear in Proc. of 4th Workshop on Nuclear Astrophysics.

Walborn, N. R., Lasker, B. M., Laidler, V. G., and Chu, Y.-H. 1987, Ap. J. Lettr., in press.

Walker, A. R. 1985, MNRAS, 212, 343.

Wood, P. R., and Faulkner, D. J. 1987, Proc. Astron. Soc. Australia, in press.

Woosley, S. E. 1987, submitted to Ap. J.

Woosley, S. E., Pinto, P. A., Martin, P., and Weaver, T. A. 1987, Ap. J., 318, 664.

Woosley, S. E., Pinto, P. A., and Ensman, L. 1988, Ap. J., 324, 000.

Woosley, S. E., and Weaver, T. A. 1987, Physics Reports, Proc. Bethe Birthday Workshop, submitted.

Woosley, S. E., and Weaver, T. A. 1986, in Radiation Hydrodynamics in Stars and Compact Objects, ed. D. Mihalas and K.-H. A. Winkler, (Springer Verlag: Berlin), p. 91. 